\title{
CONFIRMING THE PRIMARILY SMOOTH STRUCTURE OF THE VEGA DEBRIS DISK AT MILLIMETER WAVELENGTHS
}

\author{
A. Meredith Hughes ${ }^{1,11}$, David J. Wilner ${ }^{2}$, Brian Mason $^{3}$, John M. Carpenter ${ }^{4}$, Richard Plambeck ${ }^{1}$, \\ Hsin-Fang Chiang ${ }^{5,6}$, Sean M. Andrews ${ }^{2}$, Jonathan P. Williams ${ }^{7}$, Antonio Hales $^{8}, \mathrm{Kate} \mathrm{Su}^{9}$, Eugene Chiang ${ }^{1}$, \\ Simon Dicker $^{10}$, Phil Korngut $^{10}$, And Mark Devlin $^{10}$ \\ ${ }^{1}$ Department of Astronomy, University of California, Berkeley, CA 94720, USA; mhughes@ astro.berkeley.edu \\ ${ }^{2}$ Harvard-Smithsonian Center for Astrophysics, 60 Garden Street, Cambridge, MA 02138, USA \\ ${ }^{3}$ National Radio Astronomy Observatory, 520 Edgemont Road, Charlottesville, VA 22903-2475, USA \\ ${ }^{4}$ California Institute of Technology, Department of Astronomy, MC 105-24, Pasadena, CA 91125, USA \\ ${ }^{5}$ Institute for Astronomy, University of Hawaii, 640 North Aohoku Place, Hilo, HI 96720, USA \\ ${ }^{6}$ Department of Astronomy, University of Illinois at Urbana-Champaign, 1002 West Green Street, Urbana, IL 61801, USA \\ ${ }^{7}$ Institute for Astronomy, University of Hawaii, 2680 Woodlawn Drive, Honolulu, HI 96822, USA \\ ${ }^{8}$ Joint ALMA Observatory, Av. El Golf 40, Piso 18, Santiago, Chile \\ ${ }^{9}$ Steward Observatory, University of Arizona, 933 North Cherry Avenue, Tucson, AZ 85721, USA \\ ${ }^{10}$ Department of Physics and Astronomy, University of Pennsylvania, 209 South 33rd Street, Philadelphia, PA 19104, USA \\ Received 2011 November 7; accepted 2012 February 27; published 2012 April 17
}

\begin{abstract}
Clumpy structure in the debris disk around Vega has been previously reported at millimeter wavelengths and attributed to concentrations of dust grains trapped in resonances with an unseen planet. However, recent imaging at similar wavelengths with higher sensitivity has disputed the observed structure. We present three new millimeterwavelength observations that help to resolve the puzzling and contradictory observations. We have observed the Vega system with the Submillimeter Array (SMA) at a wavelength of $880 \mu \mathrm{m}$ and an angular resolution of 5"; with the Combined Array for Research in Millimeter-wave Astronomy (CARMA) at a wavelength of $1.3 \mathrm{~mm}$ and an angular resolution of 5"; and with the Green Bank Telescope (GBT) at a wavelength of $3.3 \mathrm{~mm}$ and angular resolution of $10^{\prime \prime}$. Despite high sensitivity and short baselines, we do not detect the Vega debris disk in either of the interferometric data sets (SMA and CARMA), which should be sensitive at high significance to clumpy structure based on previously reported observations. We obtain a marginal $(3 \sigma)$ detection of disk emission in the GBT data; the spatial distribution of the emission is not well constrained. We analyze the observations in the context of several different models, demonstrating that the observations are consistent with a smooth, broad, axisymmetric disk with inner radius 20-100 AU and width $\gtrsim 50 \mathrm{AU}$. The interferometric data require that at least half of the $860 \mu \mathrm{m}$ emission detected by previous single-dish observations with the James Clerk Maxwell Telescope be distributed axisymmetrically, ruling out strong contributions from flux concentrations on spatial scales of $\lesssim 100$ AU. These observations support recent results from the Plateau de Bure Interferometer indicating that previous detections of clumpy structure in the Vega debris disk were spurious.
\end{abstract}

Key words: circumstellar matter - planetary systems - planet-disk interactions - stars: individual (Vega)

Online-only material: color figures

\section{INTRODUCTION}

The presence of tenuous, second-generation dust disks around main-sequence stars came as a surprise after the launch of the Infrared Astronomical Satellite (IRAS) in 1983 (Aumann et al. 1984). A variety of physical processes is expected to remove orbiting dust grains on timescales shorter than the stellar age, primarily through collisional grinding and subsequent ejection of small grains by stellar radiation (e.g., Wyatt 2005; Strubbe \& Chiang 2006). It is therefore thought that the circumstellar dust must be regenerated, presumably through grinding collisions of planetesimals. Surveys for excess emission in the infrared have demonstrated that at least $15 \%$ of nearby main-sequence stars host debris disks (Habing et al. 2001; Bryden et al. 2006). As the sensitivity of instruments improves, that fraction is steadily increasing. Already the Herschel astronomical observatory has identified several new debris disk systems (e.g., Eiroa et al. 2010, 2011; Thompson 2010), with more likely to be announced soon as key programs wind down. Spatially resolving the emission

\footnotetext{
${ }^{11}$ Miller Fellow.
}

from the extended, faint dust disks is challenging, however, and so far only a handful of systems out of hundreds of candidates have been spatially resolved.

Resolved observations of debris disks reveal a wide range of morphologies, including broad disks, narrow rings, eccentricities, warps, and brightness asymmetries (e.g., Holland et al. 1998; Schneider et al. 1999; Wyatt et al. 1999; Heap et al. 2000; Kalas et al. 2005). Several of these morphological features have now been linked to the presence of planets in the disk; in fact, two of the three (so far) directly imaged extrasolar planetary systems were predicted based on the properties of their dust disks (Fomalhaut and $\beta$ Pictoris; Kalas et al. 2008; Lagrange et al. 2010). That said, the clumpy structure predicted to result from large dust grains tracing planetary resonances has a more checkered history. Theoretical predictions for the magnitude of dust concentration in orbital resonances vary widely, with some groups predicting a pronounced contrast in the surface density of large particles (e.g., Ozernoy et al. 2000; Wyatt 2003, hereafter W03) and others predicting very little structure even in the presence of massive planets (e.g., Kuchner \& Stark 2010). A complicating factor is that disk structure is predicted-and, 
indeed, observed - to vary strongly as a function of wavelength. For example, several debris disks have now been observed to host smooth, extended halos of small dust grains at radii far larger than the outer disk edges seen at longer wavelengths (e.g., Su et al. 2005, 2009).

Millimeter-wavelength observations are preferred for tracing dynamical interactions with planets, since they trace the largest accessible dust grain populations that are the least sensitive to the smoothing effects of stellar radiation (Wyatt 2006). Early observations were promising. The Vega debris disk, in particular, exhibited a possible azimuthal asymmetry in the first $860 \mu \mathrm{m}$ James Clerk Maxwell Telescope (JCMT) map of the disk structure (Holland et al. 1998), which was supported by interferometric observations with the Owens Valley Radio Observatory (OVRO; Koerner et al. 2001) and the Plateau de Bure Interferometer (PdBI; Wilner et al. 2002). This result was followed by other claims of clumpy structure at millimeter wavelengths, including a suggestive observation of $\epsilon$ Eridani in which the clumps appeared to move at super-Keplerian velocities between epochs, as expected for a resonant orbital configuration (Greaves et al. 2005). However, it was later pointed out that background galaxies may have contributed to the apparent asymmetry and rotation rate in $\epsilon$ Eridani (Poulton et al. 2006), and observations at a wavelength of $350 \mu \mathrm{m}$ did not confirm the presence of the $850 \mu \mathrm{m}$ clumps (Backman et al. 2009). More recently, clumpy structure has been claimed (and planetary properties inferred) in millimeter-wavelength maps of the disks around the young solar analogue HD 107146 (Corder et al. 2009) and the multiple-planet host star HR 8799 (Patience et al. 2011). Unfortunately, more sensitive followup observations did not recover the claimed clumpy structure (Hughes et al. 2011). At the time of writing of this article, the observational evidence for clumpy millimeter-wavelength structure in debris disks is shaky at best. However, it should be noted that all millimeter-wavelength observations of debris disks to date have been plagued by low signal to noise, due to the tenuous nature of the dust emission (which rarely amounts to more than an earth mass of material). It is likely that sensitive future observations, e.g., with the Atacama Large Millimeter Array (ALMA), will uncover any resonant structure at lower contrast than the all-or-nothing levels that are currently observable.

The Vega debris disk provides an interesting case-and is in many ways the prototype-of the study of clumpy structure in debris disks. The star itself is, of course, remarkably prominent both in the astronomical literature and in the night sky; it is an A0V star viewed nearly face-on to its rotation axis (Gulliver et al. 1994), located at a distance of $7.7 \mathrm{pc}$ from the Sun (Perryman et al. 1997; van Leeuwen 2007). Its age is estimated at 350 Myr (Song et al. 2000), and it is host to the first extrasolar debris disk ever observed (Aumann et al. 1984). Attempts to infer the disk size from IRAS observations resulted in estimates of 20" (150 AU; Harvey et al. 1984; van der Bliek et al. 1994); subsequent Infrared Space Observatory data suggested that the disk may be even larger, up to $36^{\prime \prime}$ (280 AU) at a wavelength of $90 \mu \mathrm{m}$ (Heinrichsen et al. 1998). The initial reconnaissance of disk morphology took place in the millimeter, with apparent clumpy structure on $9^{\prime \prime}$ (70 AU) scales attributed to the presence of an orbiting Neptune-mass planet (Holland et al. 1998; Koerner et al. 2001; Wilner et al. 2002; W03). Near-infrared interferometry with the Palomar Testbed Interferometer revealed a dust disk component much closer to the star, at a distance of only 4 AU, recently confirmed with International Occultation
Timing Association (Ciardi et al. 2001; Defrère et al. 2011). After the flurry of work on the millimeter structure, it was with some surprise that $\mathrm{Su}$ et al. (2005) reported azimuthally symmetric dust emission observed with the Spitzer Space Telescope extending out to hundreds of AU at mid-IR wavelengths. The smooth structure was confirmed by subsequent Herschel observations at wavelengths of 70-500 $\mu \mathrm{m}$; while the ring structure was resolved only at the shortest wavelengths, once again no azimuthal asymmetry was evident (Sibthorpe et al. 2010). Meanwhile, Caltech Submillimeter Observatory (CSO) observations at wavelengths of 350 and $450 \mu \mathrm{m}$ resolved the ring structure and reported tentative evidence of clumpy structure in the disk (Marsh et al. 2006). However, the position of the clumps in the CSO maps did not correspond with the clumps reported in the interferometric data; the authors suggested that perhaps the shorter-wavelength data were tracing $4: 3$ or $3: 2$ resonances rather than the 2:1 resonances detected at $860 \mu \mathrm{m}$ and $1.3 \mathrm{~mm}$ wavelengths, and that two different grain populations may be responsible for the emission. However, the interferometric images were recently called into question by Piétu et al. (2011), who re-observed Vega with PdBI in a mosaicked observation at a factor of two higher sensitivity than before and did not recover any clumpy structure.

We present three new sets of observations of the Vega system at millimeter wavelengths, with the intention of helping to resolve the puzzling and contradictory observations and models. The data were obtained with the Submillimeter Array (SMA), ${ }^{12}$ the Combined Array for Research in Millimeterwave Astronomy (CARMA), ${ }^{13}$ and the Green Bank Telescope $(\mathrm{GBT}),{ }^{14}$ at wavelengths of $880 \mu \mathrm{m}, 1.3 \mathrm{~mm}$, and $3.3 \mathrm{~mm}$, respectively. We describe the observations in Section 2, present the results in Section 3, and analyze the data in the context of several historically relevant classes of models in Section 4. We discuss the results and summarize their significance in Section 5.

\section{OBSERVATIONS}

Data were collected in a series of eight observing sessions on the GBT in spring 2009 and spring 2010. The GBTs $90 \mathrm{GHz}$ bolometer array, MUSTANG (Dicker et al. 2009) was used for these observations, giving a $9^{\prime \prime}$ FWHM beam on the sky. A total of $9.4 \mathrm{hr}$ of useful integration time were acquired on the map of Vega. A $1.5 \times 1.5$ arcmin map around Vega was obtained with alternating Lissajous patterns, some centered at the position of the star and four others offset by $\pm 15^{\prime \prime}$ in both north-south and east-west directions. Primary flux calibration was with reference to the asteroid Ceres-1, using a light curve and brightness temperature provided by T. Mueller (2011, private communication). Every 30-45 minutes the GBT beam, focus, and pointing were checked by a short ( 1 minute) observation of a compact pointing calibration source within $15^{\circ}-20^{\circ}$ of Vega.

\footnotetext{
12 The Submillimeter Array is a joint project between the Smithsonian Astrophysical Observatory and the Academia Sinica Institute of Astronomy and Astrophysics and is funded by the Smithsonian Institution and the Academia Sinica.

13 Support for CARMA construction was derived from the Gordon and Betty Moore Foundation, the Kenneth T. and Eileen L. Norris Foundation, the James $\mathrm{S}$. McDonnell Foundation, the Associates of the California Institute of Technology, the University of Chicago, the states of California, Illinois, and Maryland, and the National Science Foundation. Ongoing CARMA development and operations are supported by the National Science Foundation under a cooperative agreement, and by the CARMA partner universities. 14 The National Radio Astronomy Observatory is a facility of the National Science Foundation operated under cooperative agreement by Associated Universities, Inc.
} 
Table 1

Basic Observing Parameters

\begin{tabular}{lccc}
\hline \hline Date & 225 GHz Opacity & $\begin{array}{c}\text { Track Length } \\
(\mathrm{hr})\end{array}$ & $\begin{array}{c}\text { RMS Sensitivity }^{\mathrm{a}} \\
\left(\mathrm{mJy} \mathrm{beam}^{-1}\right)\end{array}$ \\
\hline \multicolumn{4}{c}{ 341 GHz SMA-4 GHz bandwidth } \\
\hline 2007 Apr 20 & 0.05 & 8 & 2.6 \\
2007 Apr 21 & $0.05-0.06$ & 7 & 2.6 \\
2007 Apr 22 & 0.04 & 8 & 2.2 \\
2007 Apr 23 & 0.03 & 8 & 2.0 \\
\hline \multicolumn{4}{c}{ AHz CARMA-8 GHz bandwidth } \\
\hline 2011 Jan 20 & 0.15 & 3 & 0.54 \\
2011 Jan 23 & 0.10 & 5 & 0.53 \\
2011 Jan 30 & 0.25 & 4 & 0.87 \\
2011 Feb 2 & 0.07 & 2 & 0.87 \\
2011 Feb 7 & 0.15 & 3 & 0.71 \\
2011 Feb 8 & 0.14 & 6 & 0.61 \\
2011 Feb 9 & 0.13 & 6 & 0.51 \\
\hline
\end{tabular}

Note. ${ }^{\text {a }}$ Angular resolution using natural weighting of visibilities.

Pointing corrections were applied offline in the data analysis. If the beam or focus showed evidence of drift from their optimal values, they were corrected by collecting a 15 minute outof-focus holography map, analyzing the data on the fly, and applying the corrections to the telescope (Nikolic et al. 2007). Noise levels were established by means of a suite of Monte Carlo bootstrap simulations in which the bolometer data were shifted by random amounts in time with respect to the antenna position data. Images were made by a series of IDL routines developed to analyze MUSTANG data. These routines remove a common mode from all detectors, which is highly effective at eliminating large-scale $\left(>40^{\prime \prime}\right)$ signals due to fluctuations in the atmosphere emission and cryogenic instabilities. A low-order polynomial is fitted to each detector to remove residual drifts; the individual detector weights are calculated from the rms of the residual timestream; and the resulting data gridded onto the sky. More details about the data reduction and calibration procedures can be found in Mason et al. (2010) and Dicker et al. (2009).

The SMA data were collected during four eight-hour tracks on 2007 April 20-23 in the subcompact configuration, which provided baseline lengths of 10-45 m between the eight $6 \mathrm{~m}$ antennas (see Table 1 for basic information about individual tracks). The weather was excellent, particularly on the night of April 23. The $225 \mathrm{GHz}$ opacity was stable between 0.04 and 0.06 , with the exception of a few spikes in humidity on the night of April 21. The correlator was configured for maximum continuum sensitivity, with uniform spacing of 32 channels in each $104 \mathrm{MHz}$ correlator chunk across the $2 \mathrm{GHz}$ bandwidth in each sideband. The Local Oscillator (LO) frequency was set to $340.8 \mathrm{GHz}$, placing the $\mathrm{CO}(3-2)$ line in the upper sideband. Flux calibration was carried out using Titan and MWC 349. Because of the range in right ascension covered by the target and calibrators, atmospheric and instrumental gain calibration was carried out using both MWC 349 and 3c345, with the former dominating the early part of the track and the latter the late part of the track. The derived flux of MWC 349 was $2.45 \mathrm{Jy}$; $3 \mathrm{c} 345$ varied between 2.29 and 2.51 Jy over the four nights. The quasar J1801+440 was included in the observing loop to test the quality of the phase transfer to Vega, although the photosphere of Vega was also detectable on all four nights and served as a secondary check on the calibration. Errors in the nominal baseline solutions were evident in the quasar phases, which did not track each other well. However, we were able to derive corrected baseline solutions using only the calibrators present in the data set. The updated baseline solutions resulted in a $10 \%$ improvement in signal to noise on the detection of Vega's photosphere. Routine calibration tasks were carried out using the MIR $^{15}$ software package, while imaging and deconvolution were accomplished using the MIRIAD software package. A naturally weighted image of the four data sets yields an rms noise of $0.94 \mathrm{mJy}^{\text {beam }^{-1}}$ in a $5.3 \times 4^{\prime \prime} .7$ beam at a position angle of $-80^{\circ}$.

The CARMA data were collected in a series of seven short (3-6 hr) tracks between 20 January and 2011 February 9 (see Table 1). The array was in its most compact "E" configuration, with projected baseline lengths between 5 and $65 \mathrm{~m}$. The shortest baselines were provided by the nine $6 \mathrm{~m}$ diameter antennas, while the longest baselines were preferentially between the six $10 \mathrm{~m}$ diameter antennas. The correlator was configured for maximum bandwidth and therefore continuum sensitivity, with eight $500 \mathrm{MHz}$ bands in each sideband for a total bandwidth of $8 \mathrm{GHz}$. The LO frequency was $227.5 \mathrm{GHz}$ and the IFs of the bands were distributed between 2.3 and $7.3 \mathrm{GHz}$ in such a way as to avoid atmospheric ozone lines. The observing pattern was a three-point mosaic centered on the star position and the positions of the two clumps reported in Wilner et al. (2002). Observations of Vega alternated with the quasar $\mathrm{J} 1848+323$, which was used to calibrate the atmospheric and instrumental phase variations. No test quasar was included in the observing loop since Vega's photosphere was bright enough to be detected in most of the tracks (all but the two with the poorest observing conditions) and provided an excellent test of the quality of the phase transfer. The quasar $3 \mathrm{c} 454.3$ was used as the passband calibrator, and Neptune as the flux calibrator. The derived flux of J1848+323 varied between 0.45 and $0.63 \mathrm{Jy}$, yielding a photospheric flux for Vega of approximately $0.7 \mathrm{mJy}$ beam $^{-1}$. Because Vega was a daytime source in January and the pointing on the $6 \mathrm{~m}$ antennas is poorer during the day, optical pointing was carried out every $2 \mathrm{hr}$, using the photosphere of Vega as the pointing calibrator (Corder et al. 2010). Calibration, imaging, and deconvolution were accomplished using the MIRIAD software package. The rms noise in the naturally weighted image combining all the data was $0.28 \mathrm{mJy}^{\text {beam }}{ }^{-1}$ in a $4^{\prime \prime} .2 \times 2^{\prime \prime} .5$ beam at a position angle of $-88^{\circ}$.

\section{RESULTS}

We detect the stellar photosphere in each of the three data sets, but only in the GBT data is there a marginal $(3 \sigma)$ detection of thermal emission from the dust disk. The disk emission is not detected in either of the two interferometric data sets despite lower rms noise and shorter baselines than previous observations that apparently showed clumpy dust emission.

Figure 1 shows the results of the CARMA observations. The stellar photosphere is independently detected in most of the seven short tracks, and the photospheric detection for the combined data set is at the $6 \sigma$ level. The top panel of Figure 1 shows the combined data set imaged with natural weighting, while the bottom panel shows the same visibilities imaged with a Gaussian taper to recover the lower spatial resolution with which the clumps were previously claimed to be seen. The asterisk symbols in the two panels mark the pointing centers of the three-point mosaic (with the third point located at the star position), chosen to coincide with the location of the putative

\footnotetext{
15 See http://cfa-www.harvard.edu/ cqi/mircook.html.
} 

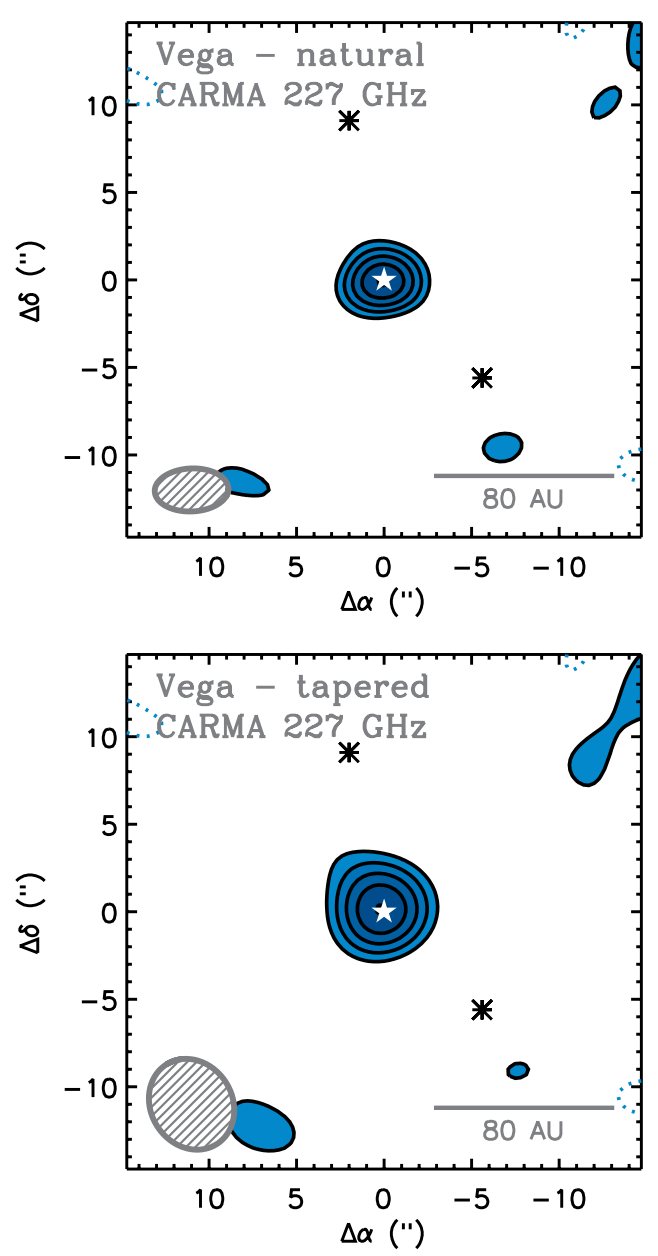

Figure 1. CARMA maps of the $227 \mathrm{GHz}$ continuum emission from the Vega system. The star symbol marks the position of Vega; thermal emission from the stellar photosphere is detected at the $6 \sigma$ level. The top panel shows an image with naturally weighted visibilities, resulting in a beam size of 4 ". $2 \times 2$ ". 5 with position angle $-88^{\circ}$. The bottom panel has been tapered in the visibility domain to match the spatial resolution of Figure 1(c) of Wilner et al. (2002), resulting in a beam size of $5^{\prime \prime} .4 \times 4^{\prime \prime} .7$ at a position angle of $32^{\circ}$. The contours are $[2,3,4,5] \times 0.28 \mathrm{mJy}$ beam $^{-1}$ (the rms noise). The asterisk symbols (along with the star symbol) mark the pointing centers of the three-point mosaic and are chosen to coincide with the position of the emission peaks reported in Wilner et al. (2002). No thermal emission from the dusty disk is detected, despite shorter baselines and lower rms noise than the PdBI data set.

(A color version of this figure is available in the online journal.)

clumps in the Wilner et al. (2002) data. No emission is detected at those locations in the CARMA data set despite a factor of two lower rms noise. The observations also have lower rms noise and richer $(u, v)$ coverage than the OVRO data obtained by Koerner et al. (2001) using the $10.4 \mathrm{~m}$ antennas that have since been incorporated into CARMA.

The SMA observations in Figure 2 tell a similar story. The photosphere is point-like, implying successful calibration of the instrumental and atmospheric gains, and is strongly detected at the $7 \sigma$ level. The higher frequency of the SMA observations requires flux scaling for comparison to the $1.3 \mathrm{~mm}$ data. Assuming a millimeter-wavelength spectral index of 2.8 for the disk, consistent with the long-wavelength Herschel fluxes reported in Sibthorpe et al. (2010), the expected integrated $880 \mu \mathrm{m}$ flux of the emission peaks reported in Wilner et al. (2002) is 20 and $12 \mathrm{mJy}$ for the northeast and southwest peak, respectively, with peak fluxes at the resolution of the data of approximately $7.4 \mathrm{mJy}$. Given the $0.9 \mathrm{mJy}$ rms noise in the

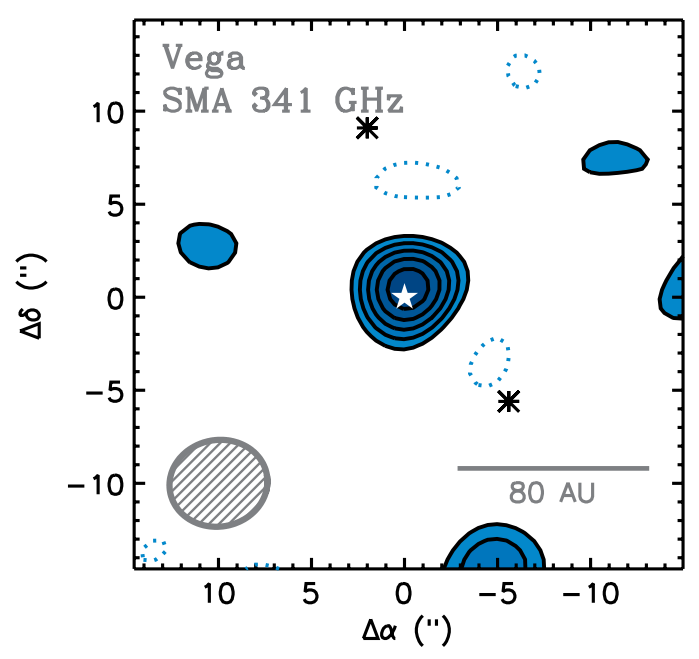

Figure 2. SMA map of the $341 \mathrm{GHz}$ continuum emission from the Vega system. Thermal emission from the stellar photosphere is detected at the $7 \sigma$ level. The visibilities have been imaged using natural weighting, resulting in a beam size of $4^{\prime \prime} .7 \times 5^{\prime \prime} .3$ with position angle $-80^{\circ}$. The contours are $[2,3,4, \ldots] \times 0.94 \mathrm{mJy}^{\text {beam }}{ }^{-1}$ (the rms noise). Symbols are as in Figure 1 . No thermal emission from the dusty disk is detected, consistent with the CARMA (Figure 1) and PdBI results (Piétu et al. 2011).

(A color version of this figure is available in the online journal.)

data, we would expect to detect emission from the clumpy dust disk at the $8 \sigma$ level (similar to the detection level of the stellar photosphere). However, no such emission was observed. This is comparable to the limits set by the CARMA data (Figure 1) and the recent updated observations from the Plateau de Bure Interferometer (Piétu et al. 2011).

Taken together, these three interferometric data sets, observed with three different instruments with broad $(u, v)$ coverage and uniformly low rms noise, place strong constraints on the amount of concentrated, clumpy emission that might be present in the Vega debris disk. They also demonstrate that previous detections of clumpy emission from the Vega system using interferometers must have been spurious. The noise may have been difficult to characterize near the edges of the primary beam, a problem mitigated in the recent data sets by the use of mosaicked imaging (CARMA, PdBI) and smaller antennas (SMA).

Given the smoothness of the disk emission indicated by the new interferometric observations, single-dish data are more desirable for characterizing the amount and location of emission from the dusty disk. The $90 \mathrm{GHz}$ GBT map is presented in Figure 3. The star position accounts for proper motion and is offset from the map pointing center by a few arcseconds. The location of the emission peak coincides with the star position, although it is only determined to within $3^{\prime \prime}$ or so due to pointing uncertainties. The map has been smoothed to $10^{\prime \prime}$ resolution (with $2^{\prime \prime}$ pixels). Because of the scanning pattern used to minimize atmospheric effects, the rms noise varies with position; it is lowest near the map center and highest at large distances from the map center. Figure 3 shows a $\pm 20^{\prime \prime}$ box around the star position. The emission peaks near the star position with a maximum signal-to-noise ratio of 4.7 . The integrated flux within a $16^{\prime \prime}$ radius of the star is $690 \pm 130 \mu \mathrm{Jy}$. To determine the expected photospheric contribution at this wavelenghth, we use the most recent Kurucz model photosphere of Vega (R. Kurucz, 2011 private communication), which is scaled to match the flux of $7.17 \mathrm{Jy}$ at $23.68 \mu \mathrm{m}$ derived by Rieke et al. (2008). We extrapolate to the millimeter regime assuming that the flux scales as $v^{2}$ in the Rayleigh-Jeans tail 


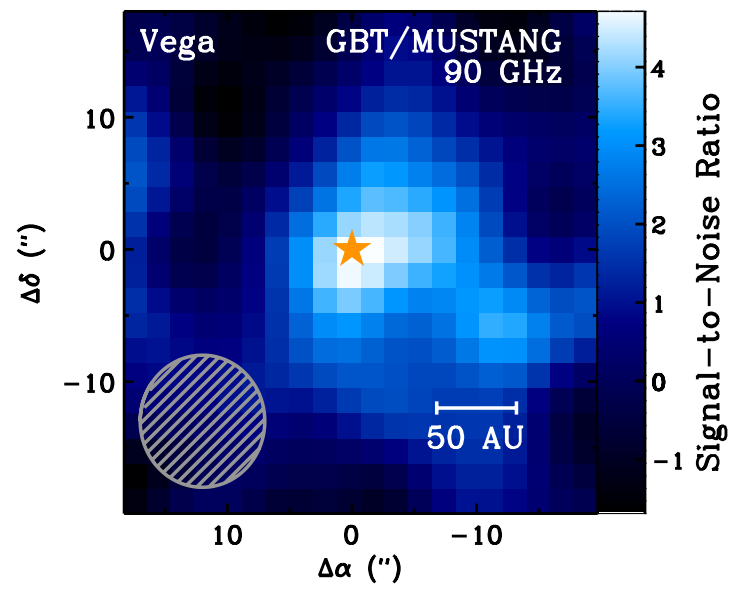

Figure 3. $90 \mathrm{GHz}$ GBT/MUSTANG map of continuum emission from the Vega system. The GBT map has been smoothed to $10^{\prime \prime}$ resolution (with $2^{\prime \prime}$ pixels), indicated by the beam in the lower left corner. The rms noise varies with position due to the scan pattern, with the lowest rms closest to the pointing center, so the data are presented as a signal-to-noise map to emphasize the significance of the emission detection as a function of position on the sky. The emission is centered on the star position (peak signal-to-noise ratio of 4.7), and the integrated flux is $690 \pm 130 \mu \mathrm{Jy}$ within a $16^{\prime \prime}$ radius from the star, indicating a marginal $(3 \sigma)$ detection of emission above the $310 \mu \mathrm{Jy}$ photosphere.

(A color version of this figure is available in the online journal.)

of the distribution. This yields photospheric fluxes of $4.4 \mathrm{mJy}$, $2.1 \mathrm{mJy}$, and $310 \mu \mathrm{Jy}$ at wavelengths of $880 \mu \mathrm{m}, 1.3 \mathrm{~mm}$, and $3.3 \mathrm{~mm}$, respectively. The GBT data therefore imply a marginal $(3 \sigma)$ detection of $375 \mu \mathrm{Jy}$ of emission above the photosphere, presumably from the dusty disk around Vega. Assuming a characteristic dust temperature of $70 \mathrm{~K}$ (Su et al. 2005, 2006) and a standard $90 \mathrm{GHz}$ dust opacity of $0.9 \mathrm{~cm}^{2} \mathrm{~g}_{\text {dust }}^{-1}$ (Beckwith et al. 1990), this corresponds to a mass of $2.3 \times 10^{-3} M_{\oplus}$, in good agreement with more detailed spectral energy distribution (SED) based models ( $\mathrm{Su}$ et al. 2005). Figure 4 plots the measured photospheric fluxes at $880 \mu \mathrm{m}$ and $1.3 \mathrm{~mm}$ along with the Kurucz model photosphere extrapolation to the millimeter spectral regime. A $3.3 \mathrm{~mm}$ upper limit $(3 \sigma)$ on the photospheric flux from Wilner et al. (2002) is also plotted. The gray shaded region of the plot represents an extrapolation of the total $860 \mu \mathrm{m}$ flux from the disk reported by Holland et al. (1998), assuming a spectral index of $2.8 \pm 0.1$ estimated from fitting the longwavelength Herschel data (Sibthorpe et al. 2010). The expected stellar contribution at $860 \mu \mathrm{m}$ is $4.4 \mathrm{mJy}$, while extrapolation of the Herschel data predict a larger $860 \mu \mathrm{m}$ flux by about the same amount (which is within the measurement uncertainty). We therefore assume that the disk flux at $860 \mu \mathrm{m}$ is $46 \mathrm{mJy}$. The GBT $3.3 \mathrm{~mm}$ flux is only marginally consistent with both the Kurucz model photosphere extrapolation and the PdBI upper limit on the photospheric flux at $3.3 \mathrm{~mm}$ (Wilner et al. 2002), supporting the conclusion that the disk contributes to the measured $3.3 \mathrm{~mm}$ flux. A substantially flatter millimeter spectral index would weaken the inferred detection of $3 \mathrm{~mm}$ emission from the Vega debris disk.

Given the low spatial resolution and signal to noise in the GBT data, it is difficult to draw strong conclusions about the spatial distribution of the emission, although the implied integrated $90 \mathrm{GHz}$ emission from the dust disk within 16" (125 AU) is approximately $375 \mu \mathrm{Jy}$. This is consistent with previous observations from the JCMT by Holland et al. (1998), who report a peak flux of $17.3 \pm 3.0 \mathrm{mJy}$ in a $14^{\prime \prime}$ beam at a wavelength of $860 \mu \mathrm{m}$. Scaling the $860 \mu \mathrm{m}$ flux to the $3.3 \mathrm{~mm}$ wavelength of

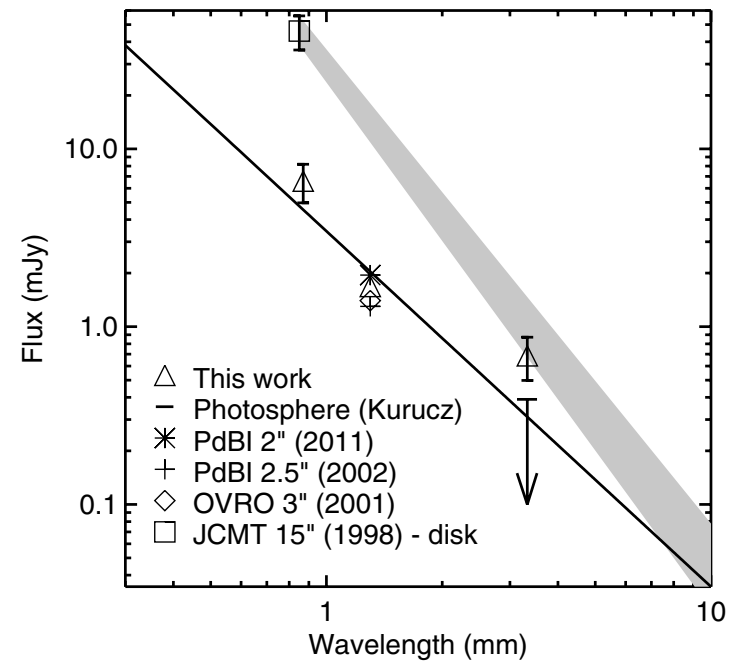

Figure 4. Millimeter-wavelength SED of Vega. Flux values from this work are plotted with triangle symbols. Error bars mark $1 \sigma$ intervals, including both the noise in the image and a $20 \%$ systematic flux uncertainty. Error bars are not included on the $1.3 \mathrm{~mm}$ data to avoid excessive cluttering around the four closely spaced points. The telescope name, angular resolution, and year of observation are indicated in the legend. Data are drawn from Holland et al. (1998), Koerner et al. (2001), Wilner et al. (2002), and Piétu et al. (2011). The disk is resolved out in the interferometric data (all points except the $860 \mu \mathrm{m}$ JCMT flux from Holland et al. 1998 and the $3.3 \mathrm{~mm}$ GBT flux), which are sensitive only to the photosphere. A $3.3 \mathrm{~mm}$ upper limit $(3 \sigma)$ on the photosphere from Wilner et al. (2002) is also included. The gray area represents an extrapolation of the $46 \pm$ $5 \mathrm{mJy}$ integrated $860 \mu \mathrm{m}$ flux measured with the JCMT (Holland et al. 1998) to longer wavelengths, assuming a spectral index of $2.8 \pm 0.1$ estimated from fitting the long-wavelength Herschel data (Sibthorpe et al. 2010). An extrapolation of the 2005 Kurucz model photosphere (solid line) is also plotted.

the GBT observations predicts $380 \mu \mathrm{Jy}$ of dust emission within $14^{\prime \prime}$ of the star, using the spectral index of $2.8 \pm 0.1$ derived from the long-wavelength Herschel data (Sibthorpe et al. 2010). The integrated emission in the JCMT map was larger, $46 \pm 5 \mathrm{mJy}$, but the increasing rms noise with distance from the GBT map center makes it difficult to detect very extended emission, so we limit ourselves to considering emission only within $\sim 125 \mathrm{AU}$ from the star. Also, given the position uncertainty in the GBT map, it is difficult to determine whether the emission peaks at the star position. The $3^{\prime \prime}$ uncertainty makes the GBT map at best marginally consistent with the $9^{\prime \prime}$ offset of the peak emission in the JCMT map; however, the photosphere represents a much larger contribution to the total flux at $3.3 \mathrm{~mm}$ than at $860 \mu \mathrm{m}$, which is likely to shift the location of the emission peak closer to the center as the disk emission is convolved with the photosphere. To summarize, while the GBT data are consistent with the JCMT map, on their own they can neither confirm nor disprove the presence of non-axisymmetric emission in the Vega debris disk.

\section{ANALYSIS}

The data presented in this paper, along with recent PdBI observations from Piétu et al. (2011), represent a marked departure from previous observations that indicated clumpy emission from the Vega debris disk. We therefore seek to characterize the parameter space consistent with the new observations. We consider three categories of models: (1) the dynamical model of a resonant planetesimal population from W03, similar to that presented in Wilner et al. (2002) and designed to reproduce the JCMT map; (2) a smooth, axisymmetric disk model, motivated by ring-like structure observed at shorter wavelengths; and (3) a 

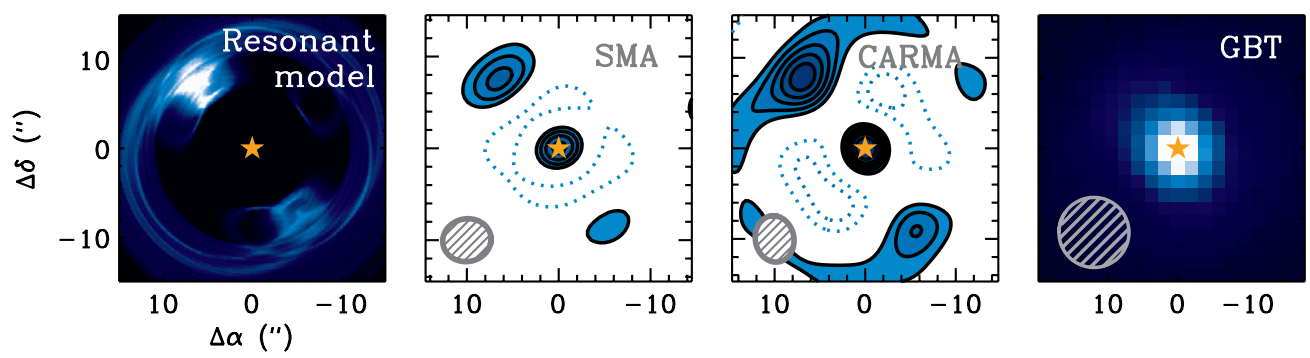

Figure 5. Simulated observations of the W03 resonant model (left) with each of the different observing setups presented in this paper. If the model were correct, we would expect to detect dust emission at the $4 \sigma$ level with the SMA at $341 \mathrm{GHz}$ (center left) and at the $6 \sigma$ level with CARMA at $227 \mathrm{GHz}$ (center right). The $90 \mathrm{GHz}$ GBT observations are consistent with the W03 model primarily because they do not have the spatial resolution or sensitivity necessary to discriminate between this and other models. The observations are simulated without noise and with identical imaging parameters and flux cutoffs to the images in Figures 1 and 2 (the negative residuals in the interferometric maps are artifacts of the CLEAN algorithm applied to the data), but the W03 model is clearly inconsistent with the observations, particularly the CARMA data. Symbols are as in Figures $1-3$, including SMA contour levels of $[2,3,4, \ldots] \times 0.94$ mJy beam $^{-1}$ and CARMA contour levels of $[2,3,4, \ldots] \times 0.28$ mJy beam $^{-1}$.

(A color version of this figure is available in the online journal.)

"toy" model of clumpy debris disk emission that can be distilled into a two-parameter space to constrain exactly how clumpy (or axisymmetric) the disk emission may be while maintaining consistency with the data. ${ }^{16}$ We compare the data with these models in sequence in the following sections. While the GBT data are not sensitive enough to strongly constrain the degree of axisymmetry of the disk emission at large distances from the star, they are useful for constraining the radial size of the flux distribution. We therefore include these data in the subsequent analysis.

\subsection{Resonant Clump Model}

Since the W03 model was generated to reproduce the clumpy emission reported in the older data sets (Holland et al. 1998; Koerner et al. 2001; Wilner et al. 2002), it is likely that it will be inconsistent with the more sensitive upper limits on clumpy disk emission from the PdBI (Piétu et al. 2011), SMA, and CARMA (this work). Nevertheless, for completeness we present a comparison of the new SMA, CARMA, and GBT data with the W03 model, scaled to the appropriate wavelengths.

Figure 5 shows a comparison of the W03 model and (noiseless) simulations of each of the three data sets presented in this paper. To simulate the interferometric observations, we sample the model at the same spatial frequencies as the data, then invert the visibilities and deconvolve the image with the same parameters as were used for the data, including the flux cutoff in the CLEAN procedure. The negative contours in the interferometric images are artifacts of the CLEAN algorithm applied to the data. The W03 model of the underlying planetesimal distribution is scaled to an appropriate flux at each wavelength. At $341 \mathrm{GHz}$, the photosphere is assumed to be $4.4 \mathrm{mJy}$ and the total flux in the disk model plus photosphere is scaled to match the integrated flux of $46 \mathrm{mJy}$ reported for the JCMT detection (Holland et al. 1998). At $227 \mathrm{GHz}$, the $850 \mu \mathrm{m}$ model is scaled in flux assuming a spectral index of 2 for the star and 2.8 for the disk emission. The $90 \mathrm{GHz}$ model prediction is generated by assuming a $310 \mu \mathrm{Jy}$ photosphere and scaling the flux within $16^{\prime \prime}$ of the star position to the integrated flux in the GBT map. The resulting simulated map demonstrates that the GBT data are not sensitive enough to rule out a resonant emission model. While the SMA data are similarly consistent with the W03 simula-

\footnotetext{
16 We also tried an eccentric ring model motivated by the double-peaked structure in the JCMT and GBT maps, but it required emission too concentrated to be consistent with the SMA and CARMA data.
}

tions, the CARMA data are clearly inconsistent with the model prediction.

\subsection{Axisymmetric Model}

Since a clumpy model is inconsistent with the observations, it is desirable to characterize the parameter space allowed for a smooth, axisymmetric model of the disk emission. A smooth model is consistent with shorter-wavelength observations of the Vega debris disk (e.g., Su et al. 2005) and with submillimeter observations from Marsh et al. (2006), and is perhaps the most natural explanation for the lack of detectable millimeterwave emission in the interferometric data sets. We therefore investigate the parameter space allowed by the combination of SMA, CARMA, and GBT data presented in this paper.

We assume a smooth, axisymmetric disk with inner radius $R_{\text {in }}$ and width $\Delta R$ (so that the disk outer radius $R_{\text {out }}=R_{\text {in }}+\Delta R$ ). We assume that the disk is viewed face-on $(i=0)$. The inclination of the stellar rotation axis to our line of sight is closer to $5^{\circ}$ (Aufdenberg et al. 2006), but the true disk inclination is not well determined. Since many previous papers modeling the Vega debris disk have assumed a face-on orientation for simplicity, we follow suit; a difference of a few degrees does not alter our conclusions. We assume constant surface density between $R_{\text {in }}$ and $R_{\text {out }}$, with temperature decreasing as $T(R) \propto R^{-0.5}$ and opacity constant with radius. Effectively, this amounts to an assumption that flux also decreases with distance from the star as $R^{-0.5}$. We make no assumptions about dust grain composition, emission or absorption efficiency, or opacity. The temperature and mass of the dust are thoroughly degenerate, and we concern ourselves only with the distribution of $f l u x$ in the disk, i.e., the product of surface density, temperature, and opacity. For standard assumptions about the dust grain composition and opacity, the temperatures assumed in our model are consistent with those in the literature (e.g., Su et al. 2005). We further adopt the observational constraints that the total flux of star and disk at $860 \mu \mathrm{m}$ is $45.7 \mathrm{mJy}$ (Holland et al. 1998); the photospheric flux at $341 \mathrm{GHz}$ is $5.7 \mathrm{mJy}$; the flux at $227 \mathrm{GHz}$ scales from $860 \mu \mathrm{m}$ with a spectral index of 2.8 ; and the total disk flux within $16^{\prime \prime}$ from the star at $90 \mathrm{GHz}$ is $375 \mu \mathrm{Jy}$. Under these constraints, we vary $R_{\text {in }}$ and $\Delta R$ to generate a grid of models to be compared with the data. For the interferometric data sets, we generate a model of the disk only (without the photosphere), and sample the visibilities with the same spatial frequencies as the observations using the MIRIAD task uvmodel. We then compare the synthetic visibilities with the observed visibilities, 


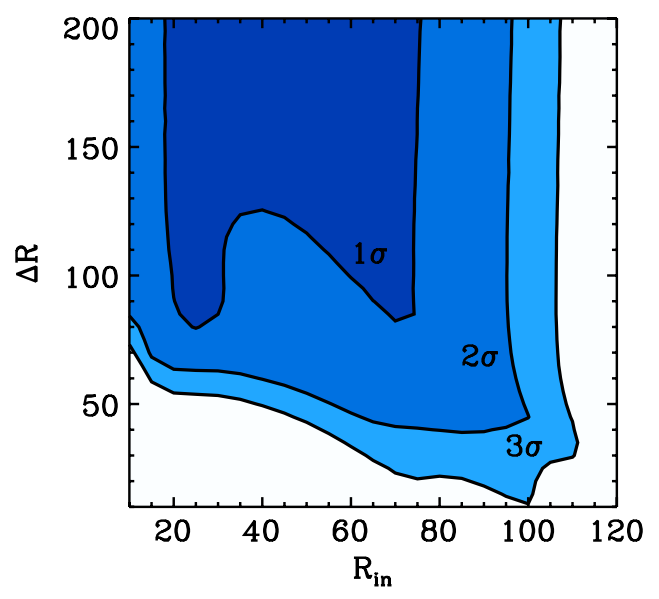

Figure 6. Confidence interval contour plot showing the regions of parameter space for a smooth disk model allowed by the combination of SMA (5" resolution), CARMA ( $\left.4^{\prime \prime}\right)$, and GBT $\left(10^{\prime \prime}\right)$ data. The abscissa corresponds to the inner radius of an axisymmetric disk while the ordinate corresponds to the width of the disk (so that the disk outer radius $R_{\text {out }}=R_{\text {in }}+\Delta R$ ). Small inner radii and small disk widths are ruled out by the interferometric data, while large inner radii are ruled out by the GBT data.

(A color version of this figure is available in the online journal.)

real and imaginary, and calculate a $\chi^{2}$ value. For the GBT data, we create a model image of the photosphere and disk, convolve it with an appropriately sized beam, and calculate the reduced $\chi^{2}$ value in a $16^{\prime \prime}$ square region around the star position, assuming the $75 \mu \mathrm{Jy}$ rms noise in the map center derived from simulated observations in Section 2.

Figure 6 presents the results of the simulations, and marks off the parameter space allowed by the SMA, CARMA, and GBT observations, given the aforementioned assumptions. Small values of $R_{\text {in }}$ and $\Delta R$ are ruled out by the interferometric data, since emission concentrated too close to the star position or in too narrow a ring around the star should have been detected in the CARMA or SMA data sets. Models with larger $R_{\text {in }}$ and $\Delta R$ exhibit smoother emission that would be undetectable by the interferometric observations; however, based on the GBT detection of $375 \mu \mathrm{m}$ within $16^{\prime \prime}(125 \mathrm{AU})$ of the star, $R_{\text {in }}$ must be smaller than this aperture. The flux distribution in the GBT data places constraints on smaller radii as well. In the context of a smooth, axisymmetric disk model, an inner radius of 20-100 AU is preferred, with a width $\gtrsim 50 \mathrm{AU}$. Inner radii $<15 \mathrm{AU}$ and $>110 \mathrm{AU}$ are ruled out by the CARMA and GBT data, respectively, while widths $<30 \mathrm{AU}$ are ruled out by the interferometric data.

\subsection{Clumpy Toy Model}

Although the data presented in Piétu et al. (2011) and this work are consistent with an azimuthally symmetric distribution of emission, it is still true that some millimeter data sets (Holland et al. 1998; Marsh et al. 2006) exhibit marginally significant evidence for a clumpy distribution of emission. In an attempt to quantify exactly how clumpy the Vega debris disk may be given the available data, we consider a toy model consisting of a smooth component underlying two Gaussian "clumps" of variable FWHM. We make several simplifying assumptions: (1) the location and relative brightness of the two clumps are fixed at $9^{\prime \prime}$ northeast and southwest of the star position, respectively, with the southwest peak 0.3 times that of the northwest peak, consistent with a literal interpretation of the JCMT data; (2) the inner and outer radius of the smooth component are fixed at

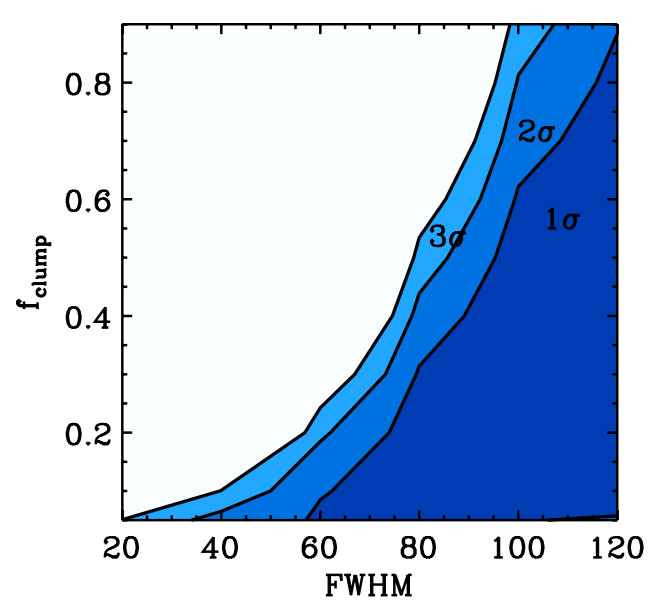

Figure 7. Confidence interval contour plot showing the regions of parameter space for a clumpy disk model allowed by the interferometric (SMA and CARMA) data. The abscissa corresponds to the FWHM of the Gaussian clumps in $\mathrm{AU}$, while the ordinate corresponds to the fraction of flux in the clumpy rather than smooth component.

(A color version of this figure is available in the online journal.)

60 and $200 \mathrm{AU}$, respectively, to maintain consistency with the results of the axisymmetric modeling from Section 4.2; and (3) the surface density of the smooth component is held constant, while the temperature is assumed to decrease with distance from the star $R$ as $R^{-1 / 2}$. As in Section 4.2, we also normalize the $880 \mu \mathrm{m}$ flux to the measured JCMT value from Holland et al. (1998), assume a spectral index of 2.8 to extrapolate to $227 \mathrm{GHz}$, and normalize the $90 \mathrm{GHz}$ disk flux to the measured $375 \mu \mathrm{Jy}$ within $125 \mathrm{AU}$ from the star. Given these assumptions, the only variables are the fraction $f_{\text {clump }}$ of total flux in the clumpy component (with the rest distributed across the smooth component), and the FWHM of the Gaussian clumps. While we do fix the position of the clumps in the disk to match the JCMT observation, the result will be more generally applicable since the interferometric data would be capable of detecting comparably clumpy structure at virtually any position in the map.

We first examine only the constraints from the interferometric data, since these data are the most sensitive to the presence or the absence of flux concentrations. Figure 7 shows the parameter space permitted by the SMA and CARMA observations: as expected, a smooth brightness distribution is favored over a clumpy distribution, although very diffuse and extended clumps (with FWHM $\gtrsim 100 \mathrm{AU}$ ) are permitted. It should be noted that the largest size scale we consider is $\sim 120 \mathrm{AU}$, since this is roughly the largest size scale to which the data are sensitive, given the $\sim 1 \mathrm{~mm}$ wavelength of observation and the $\sim 10 \mathrm{~m}$ baselines in the CARMA and SMA data. However, even when diffuse clumps are included, they should not account for more than about half of the total flux in the image to maintain consistency with the interferometric data. The constraints from the GBT data are somewhat orthogonal: due to the flux concentration in the southwest corner of the GBT map, the data favor models with a clumpy underlying flux distribution. The overlapping preferred parameter space for all three data sets occurs near the upper right corner of the plot, with large, diffuse clumps accounting for approximately half of the flux in the data, and the rest distributed in an extended smooth component. However, considering the low signal-tonoise ratio for the extended (non-photospheric) flux in the GBT map, we note that the constraints from the interferometric map 
are the strongest, and require the smooth component of the flux distribution to dominate. The CARMA and SMA therefore demonstrate that most $(\gtrsim 50 \%)$ of the flux is distributed in a smooth, axisymmetric ring around the star, although a small degree of concentration on very large scales is permitted. This result does not depend strongly on the assumed values of $R_{\text {in }}$ and $\Delta R$.

\section{DISCUSSION}

Modeling of the three new data sets favors a smooth, axisymmetric flux distribution for the Vega debris disk, with an inner radius of 20-100 AU and a broad radial width of at least $50 \mathrm{AU}$. Diffuse emission on large scales may account for up to about half of the flux, but the majority must be smoothly distributed. We have considered only a small subset of possible flux configurations for the disk, but the results are more generally applicable. In particular, the clumpy toy model demonstrates that any flux concentration on spatial scales of less than $\sim 100$ AU cannot dominate the millimeter-wavelength flux distribution. While we chose a two-clump model to approximate the morphology of the JCMT data, the result can be applied to any concentrated flux distribution. If the flux were concentrated into a single clump, for example, it would account for even less than half of the total flux. Nor is the location of the clumpy emission restrictive. The interferometric data effectively rule out clumpy emission within the $30^{\prime \prime}$ primary beam of the SMA at $880 \mu \mathrm{m}$ and within the $\sim 30^{\prime \prime} \times 45^{\prime \prime}$ approximately elliptical region covered by the three-point CARMA mosaic.

It is unlikely that the clumpy structure detected by Koerner et al. (2001) and Wilner et al. (2002) could have redistributed itself in the $\sim 10$ year interval since those observations. Resonant patterns, like those described by W03, change and move on timescales comparable to the orbital period of the planet inducing the resonance. For the putative Neptune-mass planet located $65 \mathrm{AU}$ from the star described in W03, the orbital period is approximately $300 \mathrm{yr}$. This is too long to account for a large-scale redistribution of material on a 10 year timescale (moreover, resonant structures are typically long-lived; they are not expected to dissolve on orbital timescales, much less fractions of an orbit). Stellar radiation forces can also alter the spatial distribution of dust; however, the grains responsible for the observed emission are likely millimeters in size, too large to be expelled by radiation pressure, and the Poynting-Robertson drag timescale at the 70 AU radius of the claimed clumpy structure is of the order of 1 Gyr. The dust distribution could also change because of collisions: the millimeter-sized grain population could be removed by collisional grinding or be rendered invisible by growth into larger bodies with smaller millimeterwavelength opacity. Both destruction and agglomeration occur on timescales at least as long as the collisional timescale, which can be estimated as $P_{\text {orb }} / \tau$, where $P_{\text {orb }}$ is the orbital period and $\tau$ is the vertical geometric optical depth of the disk (e.g., Lagrange et al. 2000). For a ring whose annular width is comparable to its radius, the vertical optical depth is roughly the observed ratio of infrared to bolometric luminosity, sometimes denoted $\tau_{\mathrm{IR}}$ (e.g., Backman 2004; Chiang et al. 2009). Since the Vega debris disk exhibits $\tau_{\mathrm{IR}}=2 \times 10^{-5}$ (e.g., Lagrange et al. 2000), the collision timescale must be orders of magnitude larger than the orbital timescale. Finally, one can imagine a scenario wherein the clumps observed $\sim 10$ years ago represent a transient burst of dust released from a catastrophic collision between larger parent bodies-dust whose surface brightness is today too small to be detected because of spreading of the ejecta. But even at extreme ejecta speeds of $1 \mathrm{~km} \mathrm{~s}^{-1}$, dust could only have spread across a few AU, which is an order of magnitude smaller than the linear scale corresponding to the beam size of the observations; furthermore, spreading by Keplerian differential rotation occurs over timescales at least as long as the local orbital period of $300 \mathrm{yr}$. In sum, it is difficult to imagine how the clumpy structure could have disappeared in the $\sim 10$ years that have elapsed between observations.

The interferometric data rule out millimeter-wavelength morphologies that concentrate the brightness on small scales; this includes a narrow belt or ring that would concentrate the flux radially. Ring widths of less than $30 \mathrm{AU}$ are ruled out at the $3 \sigma$ level by the interferometric data, and widths of $>100 \mathrm{AU}$ are preferred. One emerging framework for understanding debris disk structure asserts that in some systems, large particles may be concentrated into a narrow "birth ring" in which collisions generate small grains carried onto broader orbits by effects of stellar radiation (Strubbe \& Chiang 2006). This theory has had notable success in explaining the differing radial extent of millimeter-wavelength and scattered light data, for example in the disk around $\beta$ Pictoris, which exhibits a ratio $\Delta R / R$ of $<0.5$ (Wilner et al. 2011). However, the Vega debris disk is one of several for which spatially resolved millimeter data require a broad band $(\Delta R / R \gtrsim 1)$ rather than a narrow ring of flux (see also HD 107146 and HR 8799; Hughes et al. 2011). At the coarse spatial resolution and limited sensitivity of current observations, not all systems fit neatly into a birth ring paradigm. Millimeter-wavelength maps display a variety of morphologies relative to scattered light.

It should also be noted that the absence of significant clumpy structure on $\lesssim 100$ AU spatial scales does not rule out the presence of planet-mass companions. There are several relevant points to consider. The W03 model only predicts the underlying planetesimal distribution, and does not include a detailed treatment of the effects of collisions on the spatial distribution of millimeter dust grains. There is some evidence that collisional smoothing may wash out evidence of planetary resonances even for the large dust grain populations that dominate the emission at millimeter wavelengths (Kuchner \& Stark 2010), and that far more sensitive observations will be needed to observe the subtle contrast generated by dynamical interactions with orbiting planets. Furthermore, taken as a whole, the observational evidence so far points to a complex, multi-component debris disk around Vega. The data presented in this paper rule out a centrally concentrated brightness distribution (which would indeed produce a detectable flux concentration on $<100$ AU spatial scales) and instead favor an inner radius for the dust disk of 20-100 AU and a width $>50$ AU. However, the near-IR interferometry (Ciardi et al. 2001; Defrère et al. 2011) and a forthcoming re-analysis of the resolved images and SED taking into account all available mid- and far-IR data (K. Su, 2011 private communication) provide evidence for multiple dust belts in the system, including a warm asteroid belt at a radius of a few AU. The presence of multiple dust belts at different radii is suggestive of a planetary system. For example, the HR 8799 system is known to host a system of at least four planets, whose orbital radii are bracketed by warm and cold dust belts inferred from models of the system SED (Chen et al. 2009; Su et al. 2009).

The series of recent observations calling into question claims of clumpy structure in millimeter-wavelength debris disks (e.g., Piétu et al. 2011; Hughes et al. 2011, this work) also has unclear implications for future observations. While there is little doubt that debris disk structures like warps, rings, and brightness 
asymmetries are tied to the presence of planets in the disk (e.g., Wyatt et al. 1999; Heap et al. 2000), it may be necessary to achieve substantially greater sensitivity levels than initially thought to observe the density concentrations induced by orbital resonances in the large-grain dust distribution. Fortunately, such observations are rapidly becoming possible with the advent of the ALMA. The unprecedented sensitivity of ALMA combined with the recent discovery of planets orbiting in debris disks (Kalas et al. 2008; Marois et al. 2008; Lagrange et al. 2010) will provide an exciting testbed for determining the magnitude and wavelength dependence of density perturbations induced by planet-disk interactions in a range of different systems. The next-generation MUSTANG2 instrument on the GBT also promises to be a valuable addition to the suite of northernhemisphere instruments. With 25 times greater sensitivity than the current MUSTANG detector, the structure of the Vega debris disk will observable at high fidelity. MUSTANG2 will also be able to resolve a number of other nearby debris disks, opening up a new long-wavelength regime of debris disk imaging.

\section{SUMMARY}

New observations of the Vega system at a range of millimeter wavelengths support the observations of Piétu et al. (2011), indicating that previous detections of a clumpy structure in the Vega debris disk were spurious. We have analyzed new interferometric data sets from the SMA and CARMA, as well as a single-dish observation with the GBT, in the context of several models of disk structure. We find that the interferometric data in particular require a disk with a large (20-100 AU) inner radius and a broad ( $>50 \mathrm{AU}$ ) radial width. Clumpy structure on $\lesssim 100$ AU scales accounts for less than half of the total $860 \mu \mathrm{m}$ flux observed by the JCMT (Holland et al. 1998). The disk must therefore be dominated by a smooth and largely axisymmetric millimeter flux distribution.

We thank Mark Wyatt for providing us with his model of the planetesimal distribution in the Vega system. The authors also thank the MUSTANG instrument team from the University of Pennsylvania, NRAO, Cardiff University, NASA-GSFC, and NIST for their efforts on the instrument and software that have made this work possible. The GBT data were obtained under the auspices of observing program AGBT08C026. A.M.H. is supported by a fellowship from the Miller Institute for Basic Research in Science. S. Dicker is supported by NSF AST1007905. A.H. acknowledges support from Millennium Science Initiative, Chilean Ministry of Economy: Nucleus P10-022-F. E.C. acknowledges support by NSF grant AST-0909210.

\section{REFERENCES}

Aufdenberg, J. P., Mérand, A., Coudé du Foresto, V., et al. 2006, ApJ, 645, 664 Aumann, H. H., Beichman, C. A., Gillett, F. C., et al. 1984, ApJ, 278, L23 Backman, D. 2004, in ASP Conf. Ser. 324, Debris Disks and the Formation of Planets, ed. L. Caroff, L. J. Moon, D. Backman, \& E. Praton (San Francisco, CA: ASP), 9
Backman, D., Marengo, M., Stapelfeldt, K., et al. 2009, ApJ, 690, 1522 Beckwith, S. V. W., Sargent, A. I., Chini, R. S., \& Guesten, R. 1990, AJ, 99, 924

Bryden, G., Beichman, C. A., Trilling, D. E., et al. 2006, ApJ, 636, 1098

Chen, C. H., Sheehan, P., Watson, D. M., Manoj, P., \& Najita, J. R. 2009, ApJ, 701, 1367

Chiang, E., Kite, E., Kalas, P., Graham, J. R., \& Clampin, M. 2009, ApJ, 693, 734

Ciardi, D. R., van Belle, G. T., Akeson, R. L., et al. 2001, ApJ, 559, 1147

Corder, S., Carpenter, J. M., Sargent, A. I., et al. 2009, ApJ, 690, L65

Corder, S. A., Wright, M. C. H., \& Carpenter, J. M. 2010, Proc. SPIE, 7733, 15

Defrère, D., Absil, O., Augereau, J.-C., et al. 2011, A\&A, 534, A5

Dicker, S. R., Mason, B. S., Korngut, P. M., et al. 2009, ApJ, 705, 226

Eiroa, C., Fedele, D., Maldonado, J., et al. 2010, A\&A, 518, L131

Eiroa, C., Marshall, J. P., Mora, A., et al. 2011, A\&A, 536, L4

Greaves, J. S., Holland, W. S., Wyatt, M. C., et al. 2005, ApJ, 619, L187

Gulliver, A. F., Hill, G., \& Adelman, S. J. 1994, ApJ, 429, L81

Habing, H. J., Dominik, C., Jourdain de Muizon, M., et al. 2001, A\&A, 365, 545

Harvey, P. M., Wilking, B. A., \& Joy, M. 1984, Nature, 307, 441

Heap, S. R., Lindler, D. J., Lanz, T. M., et al. 2000, ApJ, 539, 435

Heinrichsen, I., Walker, H. J., \& Klaas, U. 1998, MNRAS, 293, L78

Holland, W. S., Greaves, J. S., Zuckerman, B., et al. 1998, Nature, 392, 788

Hughes, A. M., Wilner, D. J., Andrews, S. M., et al. 2011, ApJ, 740, 38

Kalas, P., Graham, J. R., Chiang, E., et al. 2008, Science, 322, 1345

Kalas, P., Graham, J. R., \& Clampin, M. 2005, Nature, 435, 1067

Koerner, D. W., Sargent, A. I., \& Ostroff, N. A. 2001, ApJ, 560, L181

Kuchner, M. J., \& Stark, C. C. 2010, AJ, 140, 1007

Lagrange, A.-M., Backman, D. E., \& Artymowicz, P. 2000, in Protostars and Planets IV, ed. V. Mannings, A. A. P. Boss, \& S. S. Russell (Tucson, AZ: Univ. Arizona Press), 639

Lagrange, A.-M., Bonnefoy, M., Chauvin, G., et al. 2010, Science, 329, 57

Marois, C., Macintosh, B., Barman, T., et al. 2008, Science, 322, 1348

Marsh, K. A., Dowell, C. D., Velusamy, T., Grogan, K., \& Beichman, C. A. 2006, ApJ, 646, L77

Mason, B. S., Dicker, S. R., Korngut, P. M., et al. 2010, ApJ, 716, 739

Nikolic, B., Prestage, R. M., Balser, D. S., Chandler, C. J., \& Hills, R. E. 2007, A\&A, 465, 685

Ozernoy, L. M., Gorkavyi, N. N., Mather, J. C., \& Taidakova, T. A. 2000, ApJ, $537, \mathrm{~L} 147$

Patience, J., Bulger, J., King, R. R., et al. 2011, A\&A, 531, L17

Perryman, M. A. C., Lindegren, L., Kovalevsky, J., et al. 1997, A\&A, 323, L49

Piétu, V., di Folco, E., Guilloteau, S., Gueth, F., \& Cox, P. 2011, A\&A, 531, L2

Poulton, C. J., Greaves, J. S., \& Collier Cameron, A. 2006, MNRAS, 372, 53

Rieke, G. H., Blaylock, M., Decin, L., et al. 2008, AJ, 135, 2245

Schneider, G., Smith, B. A., Becklin, E. E., et al. 1999, ApJ, 513, L127

Sibthorpe, B., Vandenbussche, B., Greaves, J. S., et al. 2010, A\&A, 518, L130

Song, I., Caillault, J.-P., Barrado y Navascués, D., Stauffer, J. R., \& Randich, S. 2000, ApJ, 533, L41

Strubbe, L. E., \& Chiang, E. I. 2006, ApJ, 648, 652

Su, K. Y. L., Rieke, G. H., Misselt, K. A., et al. 2005, ApJ, 628, 487

Su, K. Y. L., Rieke, G. H., Stansberry, J. A., et al. 2006, ApJ, 653, 675

Su, K. Y. L., Rieke, G. H., Stapelfeldt, K. R., et al. 2009, ApJ, 705, 314

Thompson, M. A., Smith, D. J. B., Stevens, J. A., et al. 2010, A\&A, 518, L134

van der Bliek, N. S., Prusti, T., \& Waters, L. B. F. M. 1994, A\&A, 285, 229

van Leeuwen, F. (ed.) 2007, Hipparcos, the New Reduction of the Raw Data

(Astrophysics and Space Science Library, Vol. 350; Cambridge: Cambridge Univ. Press)

Wilner, D. J., Andrews, S. M., \& Hughes, A. M. 2011, ApJ, 727, L42

Wilner, D. J., Holman, M. J., Kuchner, M. J., \& Ho, P. T. P. 2002, ApJ, 569, L115

Wyatt, M. C. 2003, ApJ, 598, 1321

Wyatt, M. C. 2005, A\&A, 433, 1007

Wyatt, M. C. 2006, ApJ, 639, 1153

Wyatt, M. C., Dermott, S. F., Telesco, C. M., et al. 1999, ApJ, 527, 918 